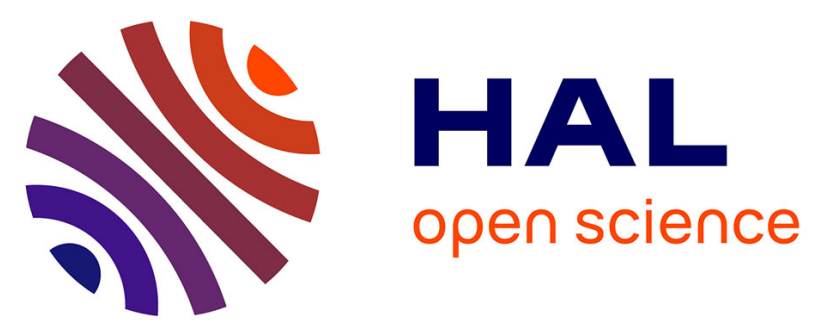

\title{
L'écrit en " représentation": les marques de grand format au sein des décors sur sigillée du Centre de la Gaule
}

Richard Delage

\section{- To cite this version:}

Richard Delage. L'écrit en "représentation ": les marques de grand format au sein des décors sur sigillée du Centre de la Gaule. Gallia - Archéologie de la France antique, 2004, L'écriture dans la société gallo-romaine, 61, pp.145-152. 10.3406/galia.2004.3191 . hal-01910994

\author{
HAL Id: hal-01910994 \\ https://hal.science/hal-01910994
}

Submitted on 29 Jan 2020

HAL is a multi-disciplinary open access archive for the deposit and dissemination of scientific research documents, whether they are published or not. The documents may come from teaching and research institutions in France or abroad, or from public or private research centers.
L'archive ouverte pluridisciplinaire $\mathbf{H A L}$, est destinée au dépôt et à la diffusion de documents scientifiques de niveau recherche, publiés ou non, émanant des établissements d'enseignement et de recherche français ou étrangers, des laboratoires publics ou privés.

\section{(ㅇ)(1) $\$$}

Distributed under a Creative Commons Attribution - NonCommercial - NoDerivatives $\mid 4.0$ 


\title{
L'ÉCRIT EN « REPRÉSENTATION »
}

\section{Les marques de grand format au sein des décors sur sigillée du Centre de la Gaule}

\author{
Richard DELAGE
}

\begin{abstract}
Mots-clés. Groupe de production céramique du Centre de la Gaule, sigillée moulée, Paternus, Cinnamus, marque épigraphique, stratégie commerciale.

Résumé. L'apparition, au sein de certains décors, à partir du milieu du If s., de marques épigraphiques de grand format constitue un des éléments de la stratégie de vente des sigillées moulées du Centre de la Gaule. L'évolution des officines majeures de Paternus et Cinnamus illustre bien ce propos.
\end{abstract}

Key-words. Central Gaul pottery production, moulded samian pottery, Paternus, Cinnamus, mark, trading strategy.

Abstract. The presence of large size stamps inside some designs from the mid $2^{\text {nd }}$ century may be considered as a feature of the sale strategy: of moulded samian pottery in Central Gaul. The evolution of the great kilns of Paternus and Cinnamus makes clear this view.

Translation: Isabelle FAUDLTT

Schlagwörter. Mittelgallische Keramikproduktion, geformte Sigillata, Paternus, Cinnamus, Inschriftenmarken, Handelsstrategie.

2 usammenfassung. Die vom 2. Jahrh. n. Chr. an in bestimmten Dekorsystemen von Sigillatagefäßen erscheinenden großen

Inschriftenmarken gehören bei der mittelgallischen Formschüsselware zur Verkaufsstrategie. Die Entwicklung der Großbetriebe des Paternus 1 nd des Cinnamus bestätigt diese Aussage.

Übersetzung : Stefan WIRTH

Les potiers de la vallée de l'Allier, et plus particulièrement ceux de Lezoux, ont eu fréquemment recours à l'écrit dans le cadre de leur activité professionnelle. Ils ont apposé sur leurs vases des estampilles ou encore inscrit leur nom, ainsi que diverses formules, dans l'argile des outils de production. À l'image des autres grands centres de production de céramique sigillée de Gaule, les pratiques adoptées par les artisans sont très variables et, suivant le support, la période ou les officines en cause, elles peuvent relever de décisions collectives ou, à l'inverse, de choix individuels. Dans tous les cas, l'analyse de cette question, relativement complexe, aboutit globalement aux mêmes conclusions que celles proposées par C. Bémont, dans ce dossier (p. 103-131), concernant le centre de production de La Graufesenque à Millau : les écrits ont essentiellement une fonction liée à l'organisation des ateliers de potiers. Ils permettent à différents intervenants de contrôler et vraisemblablement rémunérer le travail, ils servent d'aide-mémoire, de marqueurs de propriété et peuvent être le reflet d'une certaine forme de hiérarchie au sein des officines. C'est pour cela qu'ils se présentent, presque toujours, sous des formes simples, souvent abrégées voire, pour un petit nombre d'entre eux, inintelligibles.

Les ateliers du Centre de la Gaule sont à l'origine, toutefois, d'exceptions remarquables qui se distinguent des précédents par leur présentation, les modalités de leur insertion au sein des décors et, bien évidemment, par la finalité de leur conception : il s'agit de réelles tentatives de se servir des marques de potiers comme éléments de renommée favorisant le commerce ${ }^{248}$.

248. Ne sont pas pris en compte, parmi ces " exceptions ", les quelques écrits qui se démarquent radicalement des estampilles ou graffites de potiers par leur libellé et leur mise en scène et qui ne jouent aucun rôle dans l'organisation interne des ateliers. Il s'agit, pour le groupe du Centre de la Gaule, de courts textes tracés à la main dans l'argile fraiche des moules de Cirnnamus (Déchelette, 1904, t. I, p. 217 sqq. ; voir en dernier lieu : Lambert, 2002, L-70, p. 185 sqq.). Ils occupent une des métopes de la composition, sont inscrits sur plusieurs lignes, en cursive assez peu soignée, et demeurent en grande partie difficiles à interpréter. Les textes d'autres ateliers, appartenant à cette catégorie, sont bien plus célèbres. Il s'agit notamment de légendes de scènes mythologiques ou historiques présentes sur les vases des ateliers de La Graufesenque à Millau (Hedan, Vernhet, 1974 ; Mees, 1995, pl. 34-35 et 58) ou de Rheinzabern (Ricken, 1948, pl. 64), mais aussi d'apostrophes et de dédicaces à des peuples gaulois prenant place sur de rares vases moulés de Banassac (Hofmann, 1988, pl. 50-56). Tous présentent un caractère exceptionnel, dans la mesure où ils constituent une goutte d'eau dans un océan de lettres. Plus que le reflet d'une véritable politique commerciale visant à faciliter la vente, ils sont issus de l'initiative de quelques potiers, qui ne débouche guère sur des pratiques collectives. 
Les officines ayant le plus mis en œuvre ces nouvelles pratiques, bien que peu nombreuses, constituent les plus grosses unités de production du II $^{\mathbf{e}} \mathbf{s}$., celles qui contribuent à la création d'un réseau étroit de relations entre les différents centres de productions implantés le long de l'Allier, celles, encore, qui permettent aux vaisselles arvernes de conquérir les marchés à longue distance au cours du $\mathrm{II}^{\mathrm{e}} \mathrm{s}$. Cette initiative s'inscrit, ainsi, au sein d'une stratégie commerciale globale qui vise à se servir de différents atouts pour asseoir son emprise sur des marchés très concurrentiels.

Il va de soi que, dans le cadre d'un dossier comme celui-ci et compte tenu du caractère relativement pointu de cette question, nous ne pouvons présenter les tenants et aboutissants que dans leurs grandes lignes. D'autres études parues ou à paraître présentent dans le détail l'ensemble des arguments sur lesquels s'appuie cette démonstration, mais également la vision économique qu'elle implique ${ }^{249}$.

\section{LES MUTATIONS DU II' S. AU SEIN DES ATELIERS DU CENTRE DE LA GAULE}

Avant d'aborder l'étude proprement dite des deux groupes d'artisans décorateurs pris en exemple (groupes Cinnamus et Paternus), il n'est pas inutile de revenir sur quelques notions permettant de situer le contexte dans lequel ils apparaissent et se développent, ainsi que sur les aspects essentiels de l'évolution des marques épigraphiques et de leur usage sur sigillée moulée.

249. L'analyse de ces marques ne constitue qu'un des aspects d'un domaine d'étude relativement complexe : celui visant à appréhender, dans sa globalité, la chaîne opératoire de production/commercialisation/utilisation d'un des biens de consommation les plus courants du monde romain occidental. Pour arriver à cerner ne serait-ce qu'une ébauche des mécanismes qui la régissent, il est nécessaire de prendre en compte les données archéologiques dans toute leur diversité et de les interpréter en faisant preuve d'ouverture d'esprit. Nombreuses sont désormais les approches qui tendent à remettre en cause une vision systématiquement " primitiviste » de l'économie romaine, en montrant que certains secteurs artisanaux ou marchands recherchent des solutions adaptées à des situations particulières de concurrence, dans le but de pérenniser l'activité, mais aussi d'en tirer des profits. La céramique sigillée est un des exemples archéologiques les plus éloquents (voir parmi les études récentes : Dark, 2001, p. 22). Il ne s'agit pas de revenir à des interprétations de type "moderniste ", tout aussi manichéennes que les précédentes, mais plutôt de mettre au centre des débats la capacité d'adaptation de tous les intervenants de la chaîne opératoire (et non pas seulement des potiers), le bien fondé des notions de concurrence, de choix techniques et organisationnels subordonnés aux choix commerciaux. L'usage, dans cet article, de certains termes rendant compte de ces propositions peut sembler abusif tant ce domaine de recherche reste encore " sensible ". Pourtant, il n'était pas question de contourner le problème en usant de périphrases qui auraient alourdi considérablement un propos déjà dense. Évoquer les termes de "part de marché ", de "stratégies commerciales " ne rend pas compte de l'adoption aveugle d'une théorie économique au profit d'une autre, mais plutôt de phénomènes que de nombreux archéologues travaillant sur les sigillées du monde romain ont pu observer et qu'ils s'évertuent depuis plus d'un siècle à décrypter avec plus ou moins de pertinence.
Le II"s. est la période où se multiplient, au sein de la vallée de l'Allier, les ateliers de potiers fabriquant des céramiques sigillées. L'adoption des techniques de tradition méditerranéenne pour la conception de ces vaisselles de table populaires et l'ouverture des marchés d'Occident romain constituent deux des facteurs clés à l'origine de ce développement. Les différentes analyses menées depuis de nombreuses années sur l'évolution de la diffusion des vases, et aussi sur les choix en matière de production (caractéristiques techniques, répertoire morphologique, décors, etc.) et de mutation des infrastructures de fabrication, permettent d'établir aujourd'hui un panorama relativement précis de ce phénomène. On sait, de la sorte, que les territoires approvisionnés sont de plus en plus nombreux au cours de la première moitié du II $^{\mathrm{C}} \mathrm{s}$. et que l'emprise de la concurrence est de plus en plus faible au sein de la plupart d'entre eux. La couverture maximale est atteinte au milieu du II $^{\mathrm{r}} \mathrm{s}$, , puis la zone commerciale régresse ensuite, inexorablement, pour se concentrer finalement au III ${ }^{\mathrm{e}}$ s. sur le centre et l'ouest de la Gaule.

Au cours de la phase d'expansion (première moitié du II"s.), les ateliers de potiers adoptent, progressivement, une organisation du travail à caractère " collectif ", mettent en commun certaines étapes de la chaîne opératoire, répondent à des cahiers des charges relevant de contraintes commerciales et forment de nouvelles générations d'artisans qui prennent l'habitude d'évoluer dans un environnement de plus en plus complexe. Chacun ne maîtrise plus alors, bien souvent, qu'une faible part de l'ensemble des tâches dévolues à une unité de production familiale, afin d'accroître les rendements et de rendre possible l'approvisionnement de chaque foyer en vaisselle de table (Delage, 1998, 1999a et 2001 ; Picon, 2002).

L'écrit n'échappe pas à ce tourbillon de nouveautés et les coutumes liées à son usage, tout en s'inscrivant dans la continuité de ce qu'elles furent par le passé, répondent aux nécessités du commerce à longue distance ainsi qu'aux nouvelles pratiques organisationnelles. Les marques de potiers sur sigillée moulée, leur nature et leur usage constituent de bonnes illustrations de ces propos.

\section{DES PRATIQUES QUI ÉVOLUENT}

Au I ${ }^{\mathrm{cr}}$ s., le nombre d'écrits liés à la phase de fabrication du décor est très faible au sein des ateliers lézoviens. En cela, ils ne se distinguent guère de leurs homologues rutènes (voir l'analyse de C. Bémont, supra, p. 103-131). Durant toute la période où les vases carénés (forme Drag. 29) dominent le répertoire des productions moulées, à savoir jusqu'à l'ère flavienne, les marques sont apposées, pour l'essentiel, lors de la phase de fabrication du vase par le potier tourneur ${ }^{250}$. Lorsque les artisans décident d'abandonner ces coupes de forme " complexe " et de les remplacer par des vases hémisphériques de profil plus simple

250. Sur les sigillées moulées de L.czoux produites au $\mathrm{I}^{\mathrm{er}} \mathrm{s}$., à peinc plus d'une dizaine de marques in forma a pu être recensée. A La Graufesenque (Millau), elles sont plus nombreuses, mais représentent tout de même un corpus secondaire en comparaison de celles apposées sur le fond interne (Bet, Delage, 1991; Mees, 1994 et 1995). 
(forme Drag. 37), cette tendance s'inverse. À partir des dernières décennies du $\mathrm{I}^{\mathrm{rT}} \mathrm{s}$., la maîtrise et l'usage de l'écrit passent ainsi progressivement des mains du potier tourneur de vases ornés à celles du potier décorateur ou du responsable de l'officine créatrice des moules ${ }^{251}$. Nombre d'entre eux se munissent de poinçons ou inscrivent leur nom dans l'argile crue des matrices à l'aide d'un stylet. On assiste ainsi à une multiplication des marques différentes, mais également à une hausse considérable de la fréquence d'apparition de celles-ci.

Ces changements constituent quelques-uns des indices témoignant d'une évolution significative de l'organisation du travail et des priorités de production. Ils vont également progressivement suggérer à des potiers, ou à d'autres intervenants non impliqués directement dans la production, l'idée que les marques peuvent avoir une autre fonction que celle de contribuer à l'organisation des ateliers, à savoir un rôle à jouer dans la stratégie commerciale. Pour cela, elles vont s'affranchir du format relativement modeste qui fut le leur pendant des décennies et qu'elles conservent encore majoritairement au cours de la première moitié du II"s. (moins de $30 \mathrm{~mm}$ de long et $4 \mathrm{~mm}$ de haut pour les estampilles). Celui-ci, en effet, ne leur permet pas d'être de véritables "repères ", tant, la plupart du temps, il faut les chercher pour les apercevoir. Par ailleurs, la relation de la marque au décor va évoluer. $\mathrm{D}$ ' “ intruse " au sein de la composition décorative, elle devient à partir du milieu du II $^{\mathrm{e}}$ s. l'un des éléments du décor (lorsque son emplacement est réservé), ou bien elle s'impose à lui en masquant un ou plusieurs poinçons (lorsqu'elle est apposée en phase finale). Dans tous les cas, l'objectif est qu'elle puisse être lue ou tout au moins identifiée visuellement ${ }^{252}$.

Deux exemples, choisis parmi les officines majeures du Centre de la Gaule au II $^{\circ}$ s., mettent clairement en évidence cette évolution : Paternus et Cinnamus. Si leurs débuts ne se démarquent en rien de la majorité des autres ateliers lézoviens,

251. Notons, toutefois, que les marques des potiers tourneurs ne disparaissent pas complètement. Si elles ne sont plus apposées sur le fond interne des vases, elles figurent encore, en petit nombre, sur la partie externe, soit sous le décor (majoritairement des graffites), soit sur le bandeau au-dessus de celui-ci (exclusivement des estampilles). Aux II ${ }^{*}$ et III" s., ces pratiques ne sont pas systématiques. Les potiers de certains centres de production de Gaule et de Germanic scmblent y avoir recours régulièrement : Toulon-sur-Allier, Lubié à Lapalisse, Rheinzabern, etc. D'autres, en revanche, sauf exceptions, les ignorent : Les Martres-de-Veyre, Lezoux, etc. (Bet, Delage, 1991, p. 194 ; Bémont, 1997 ; Delage, 1999b, p. 315).

252. Sur les sigillées moulées de Le\%oux, alors que les estampilles de petit format (longueur inférieure en moyenne à $30 \mathrm{~mm}$ ) sont majoritairement rétrogrades, celles de plus grande taille comptent plus d'exemplaires antégrades (sens de lecture normal) que l'inverse. Ces dernières ont donc été conçues pour être lues sur les vases moulés (Bet, Delage, 1991, fig. 4). Les graffites, quant à eux, demeurent majoritairement rétrogrades. Cela est dû au fait que les potiers ne maitrisent pas l'écriture automatique à l'envers lorsqu'ils inscrivent leur nom dans le moule. En revanche, lorsqu'ils conçoivent leurs poinçons, ils peuvent prendre le temps de tracer les lettres sans commettre d'erreur. Précisons que, dans tous les cas, l'analyse quantitative des marques antégrades ou rétrogrades n'offre jamais de résultats très tranchés. Seules des tendances peuvent être dégagées, c'est-à-dire des pistes de réflexion ou des éléments de validation d'une hypothèse. elles connaissent en quelques décennics un développement sans précédent de leur activité puisque, dès le milieu ou le troisième quart du $\mathrm{Ir}^{*}$ s., les vases moulés portant des décors créés par les potiers de ces entités constituent l'essentiel des pièces diffusées à longue distance ${ }^{253}$. Cela est dû non seulement à la présence de nombreux artisans spécialisés, mais aussi au fait qu'il existe un véritable réseau de distribution de moules, en grande partie alimenté par elles, au sein duquel se trouvent impliquées des dizaines d'officines concevant des vases moulés. Les groupes Paternus et Cinnamus possèdent, ainsi, un véritable pouvoir économique qui pèse considérablement sur les choix opérés lors de la distribution des vases. Plus que d'autres, c'est l'étude de ces ensembles qui permet aujourd'hui de mettre en évidence l'implication de l'écrit dans les stratégies commerciales.

\section{L'APPARITION DES MARQUES DE GRAND FOMAT : EXEMPLES DE PATERNVS ET CINNAMVS}

Les premières marques au nom de Paternus apparaissent au cours des années 140. Ce sont des estampilles de format équivalent à toutes celles utilisées sur sigillée lisse et moulée jusqu'à cette date : moins de $30 \mathrm{~mm}$ de long ${ }^{254}$ (fig. $77 \mathrm{a}, \mathrm{n}^{\circ} \mathrm{l}$ ). Elles sont associées à un groupe de décors qui compte également d'autres petites estampilles. Parmi celles-ci figure le nom $\mathrm{du}$ potier Carantinus (fig. $77 \mathrm{~b}, \mathrm{n}^{\circ} 2$ ), ainsi qu'une marque d'officine au nom de Ianuaris (fig. $77 \mathrm{c}, \mathrm{n}^{\circ} 3$ ). Il est probable, de ce fait, que Paternus et Carantinus soient deux décorateurs

253. Quelques exemples d'études de lots de céramique sigillée issus des provinces danubiennes suffisent à le démontrer. Sur le site d'Ovilava, P. Karnitsch a inventorié, dans son étude de 1959, un peu plus de 320 décors du Centre de la Gaule, dont $30 \%$ sont liés au groupe Cinnamus et $26 \%$ au groupe Paternus (Karnitsch, 1959). Sur celui de Lentia/Lintz, les pourcentages sont cncore plus importants : $37 \%$ pour le premier et $30 \%$ pour le second (sur un total de 62 décors, voir Karnitsch, 1972). Sur les 412 compositions de la vallée de l'Allier issues des fouilles de VindobonaNienne, publices récemment par I. Weber-Hiden, $15,5 \%$ sont attribuables au groupe Paternus et pas moins de 40,8\% au groupe Cinnamus (Weber-Hiden, 1996). En Dacic, un recensement établi, il y a quelques années, par G. Popilian permettait à l'auteur de conclure : " sur les 41 fragments présentés, 18 proviemnent des atcliers de la Gaule Centrale. Comme d'habitude, les potiers les micux représentés sont Paternus et Cinnamus [...]. " (Popilian, 1977, p. 343). Un inventaire des céramiques sigillées d'Olténie montre que près de $17 \%$ des vaisselles moulées ont un décor lié au groupe Paternus, ce qui ne représente pas moins de $28 \%$ de l'ensemble des vases du Centre de la Gaule (Popilian, 1973, p. 184). Tel est également le cas des pièces du groupe Cinnamus. Même en dehors de l'Empire, parmi les rares fragments de vases moulés du Centre de la Gaule mis au jour sur le territoire de la Pologne (presque 30 pièces), les compositions de Cinnamus et Paternus représentent plus de $50 \%$ des occurrences (Tyszler, 1999).

254. Traditionnellement, ces décors précoces sont regroupés sous la mention "Paternus I ", datés par J. A. Stanfield et G. Simpson des années 150-170 et par G. B. Rogers des années 1350-160. De nouvelles analyses, fondées sur une approche méthodologique plus rationnelle, ont rendu toutefois ce classement caduc (Delage, 1999a et b). 
M1hista9

b MIGITИกภAJ

c orbaunal

d EगDVTXA

e GADVTAA

$\$ 11 / 2779$

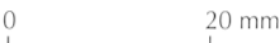

श्याज्याजए
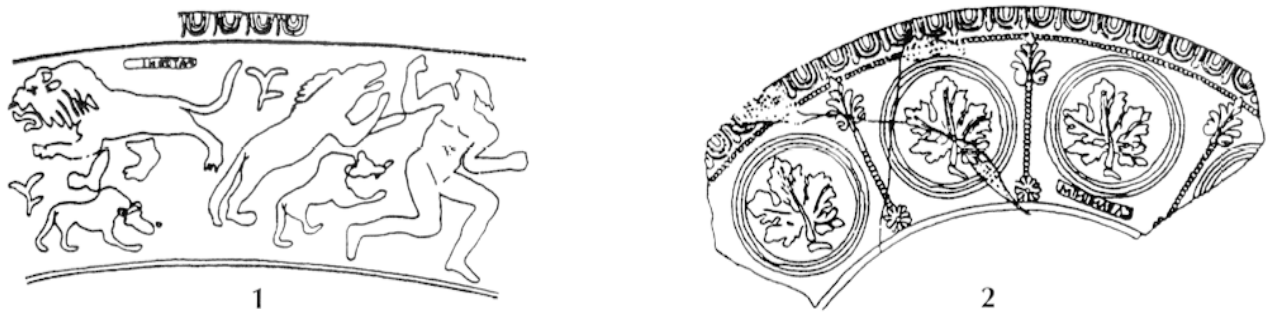

2
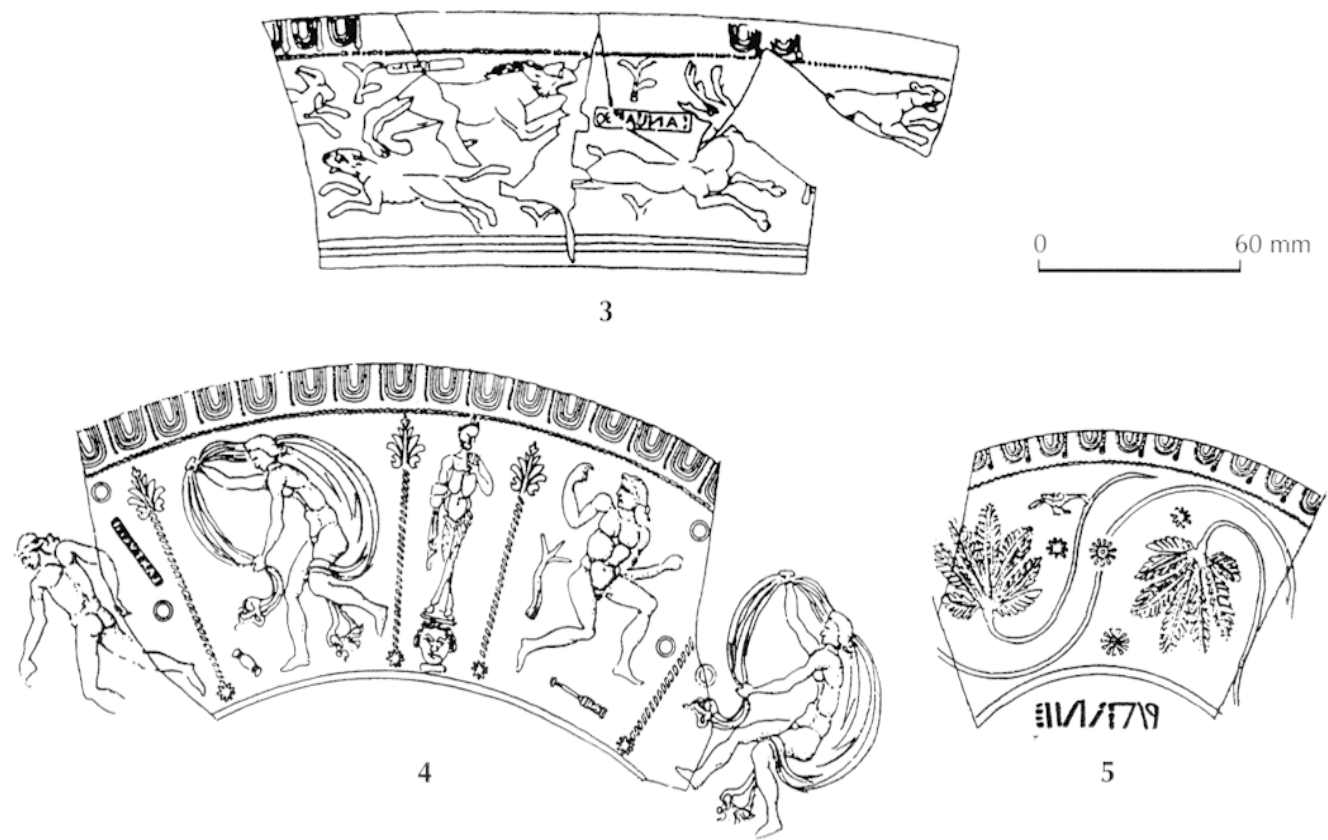

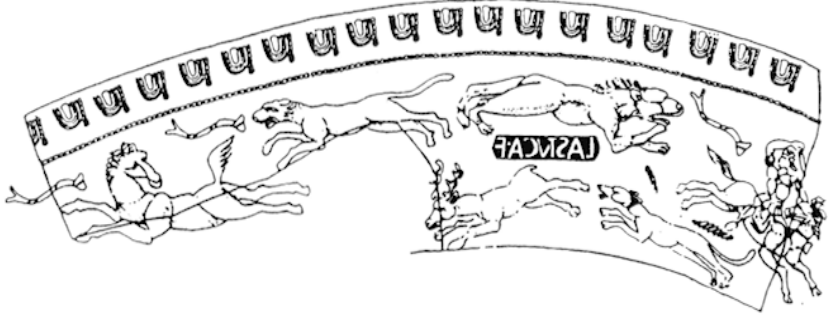

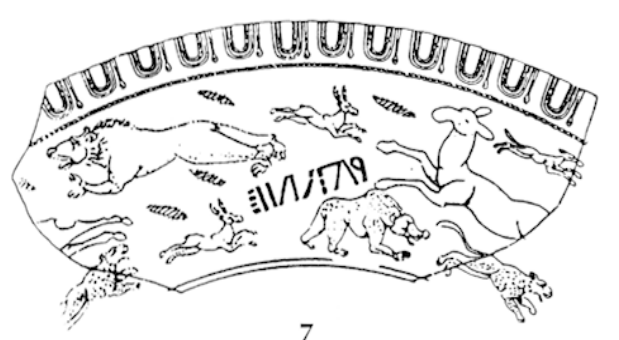

Fig. 77 - Marques intradécoratives (a à f) et décors avec marque épigraphique du groupe Paternus : a, estampille rétrograde de graphie PATERN.IM ; $\boldsymbol{b}$, estampille rétrograde de graphie CARANTINIM $; \boldsymbol{c}$, estampille rétrograde de graphie IANVARISO; $\boldsymbol{d}$, estampille rétrograde de graphie LAXTVCISF ; $\boldsymbol{e}$, estampille vétrograde de graphie LASTVCA.F; f, estampille rétrograde de graphie PATER NFE ; 1 , vase moulé comportant une estampille intradécorative rétrograde de graphie PATERN.I[M] (estampille a), provenance inconnue, lieu de conservation: musée d'Angers (Rogers, 1999, pl. 51, $n^{\prime \prime}$ 7) ; 2, vase moulé comportant une estampille intradécorative rétrograde de graphie CARANTINIM (estampille b), provenance: Wels, Haute Autriche (Karnitsch, 1959, pl. 61, $n^{n} 8$ ) ; 3, vase moulé comportant une estampille intradécorative rétrograde de graphie IANVA[RI]SO (estampille c) ainsi qu'une estampille intradécorative rétrograde de graphie [PAT]ERN.IM (estampille a), provenance: Thésé, Loir-et-Cher (Thion, 1982, fig. 59, $\left.n^{\prime \prime} 108\right) ; 4$, vase moulé comportant une estampille intradécorative rétrograde de graphie LAXTVCISF (estampille d), provenance : Lezoux, Iuy-de-Dôme (Delage, 1999a, pl. PAT-3, inv. AF015) ; 5, vase moulé comportanı une estampille infradécorative rétrograde de graphie PATER $\overline{F E}$ (estampille f), provenance : Kérilien, commune de Plounéventer, Fïnistère (Delage, 1999a, pl. PAT-5, inv. BK252) ; 6, vase moulé comportant une estampille intradécorative rétrograde de graphie LASTVCA.F (estampille e), provenance: Kérilien, commune de Plounéventer, Finistère (Delage, 1999a, pl. PAT-22, inv. BK085) ; 7, vase moulé comportant une estampille intradécorative rétrograde de graphie PATER $N \overline{F E}$ (estampille f), provenance : Lezoux, Puy-de-Dôme (Delage, 1999a, pl. PAT-42, inv. 3064). 
travaillant pour ou avec un certain Ianuaris, possesseur ou responsable de l'atelier. Notons que l'estampille PATERNVS est fréquemment associée, sur un même décor, à cette marque d'officine (fig. $77, \mathrm{n}^{\circ} 3$ ). Un certain nombre d'indices, mis en évidence notamment à partir d'autres cas d'associations, pourrait indiquer que Paternus ait eu, à cette période, un statut provisoire (apprentissage par exemple) ou bien encore qu'il ait pu débuter dans sa fonction. Durant cette première phase de travail apparaît, vraisemblablement, quelque temps après les autres, une quatrième signature au nom de Laxtucissa (fig. 77e, $\mathrm{n}^{\circ} 6$ ). Elle est la seule, avec Paternus, à perdurer au cours de la seconde phase.

Celle-ci débute vers les années 150-160. Paternus abandonne alors la petite estampille au profit d'une marque de taille plus importante, dépourvue de cartouche, de graphie PATER $\overline{\mathrm{FE}}$ : $36 \mathrm{~mm}$ de long pour $9 \mathrm{~mm}$ de haut (fig. $77 \mathrm{f}, \mathrm{n}^{\circ} 7$ ). Il est probable que les deux potiers (Paternus et Laxtucissa), associés, soient désormais maîtres de leur destin et dirigent alors leur propre officine. Durant les premiers temps de cette seconde phase, un fait intéressant est à noter : alors que Laxtucissa appose sa marque, longue de $25 \mathrm{~mm}$, dans le décor, Paternus ne le fait que sous celui-ci (fig. $77, \mathrm{n}^{\circ} 5$ ). Très vite, toutefois, cette dernière trouve également une place au sein de la partie moulée (vraisemblablement à partir des années 160). Parallèlement, en remplacement de l'estampille LAXTVCISSA, apparaît une signature de graphie LASTVCAF (fig. 77e, $\mathrm{n}^{\circ} 6$ ), de plus grande taille que cette dernière $(32 \mathrm{~mm})$, sans atteindre toutefois les dimensions du poinçon au nom de Paternus (36 mm).

Débute alors la troisième phase de l'activité de l'officine, que l'on peut situer après 170. Elle se caractérise par des volumes de production considérables. La (ou les) officine (s) correspondante(s) compte (nt) désormais un personnel nombreux, dont les individualités se fondent au sein d'une organisation nécessairement très structurée. Aucune nouvelle marque prépondérante n'apparaît à partir de cette période et l'estampille de Paternus représente la presque totalité du corpus épigraphique ${ }^{255}$. Tous les artisans décorateurs - et il ne fait aucun doute qu'ils sont nombreux ${ }^{256}$ - œuvrent ainsi, avant tout, collectivement.

Le (ou les) atelier(s) dit(s) de Cinnamus constitue(nt) une entité de production de moules tout aussi importante que celle de Paternus, si ce n'est plus. Aussi, les données dont on dispose pour établir une évolution précise ne manquent-elles pas. Elles n'ont toutefois pas encore pu bénéficier d'une analyse similaire à celle menée sur le groupe précédent ${ }^{257}$, ce qui implique une moins grande précision et, surtout, des hypothèses dont les fondements restent en grande partie à valider. Les quelques études publiées jusqu'à présent, notamment celle rédigée par A.-M. Romeuf dans sa thèse, permettent tout de même de dégager l'essentiel des informations nécessaires à cette démonstration (Vialatte, 1968 [thèse de A.-M. Romeuf] ; Simpson, Rogers, 1969 ; Romeuf-Vialatte, 1970 ; Rogers, 1972 ; Stanfield, Simpson, 1990, p. 303 sqq. ; Romeuf, Romeuf, 2001, p. 113 sqq.).

Les premières productions du groupe Cinnamus apparaissent vers 130-140 258. Elles comportent deux types de marques de décorateur : des petites estampilles et des marques cursives. Le corpus des premières, longues de 23 à $28 \mathrm{~mm}$, est riche de plusieurs poinçons différents au nom de Cinnamus (fig. 78a-d, $\mathrm{n}^{\circ} 1$ ) et un seul au nom de Paullus (fig. 78, $\mathrm{n}^{\circ} 2$ ). celui des secondes, longues de 40 à $75 \mathrm{~mm}$, comporte les noms Cinnamus (fig. 78, n⿳3), Galenus (fig. 78, n⿳4) (Romeuf, Romeuf, 2001, p. 122 sqq.) et Cerialis (fig. 78, $\mathrm{n}^{\circ} 5$ ).

Une marque d'officine au nom de Sacer ${ }^{259}$ complète cet ensemble (fig. 78, $n^{\circ} 6$ ). Notons, par ailleurs, qu'un petit nombre de vases moulés comporte deux de ces marques en association, notamment la petite estampille CINNAMI et la cursive Cerialis, confirmant le fait qu'ils œuvrent bien, à la même période, au sein de cette officine (Knorr, 1921, fig. 4, $\mathrm{n}^{\circ} 4$ ).

Grâce à la richesse du corpus épigraphique, cette première phase d'activité se révèle ainsi être complexe. Des constantes, particulièrement intéressantes, peuvent être mises en avant. Les estampilles sont exclusivement présentes dans le décor, à l'exception de celles d'officine et de quelques exemplaires de Paullus, tandis que les marques cursives figurent exclusivement sous le décor. Si ces pratiques sont probablement le reflet de coutumes liées à la position professionnelle des signataires, elles mettent aussi clairement en évidence le fait que, pour les acteurs de cette officine, à cette période, seules des marques de petite taille peuvent prendre place au sein de la composition. L'exclusion de la marque OFISACER, de plus grandes dimensions que les autres, ne fait que le confirmer.

Après les années 150 se met en place une nouvelle organisation. La plupart des noms impliqués dans la première phase d'activité n'est plus mentionnée. Cinnamus, qui devait être précédemment un des principaux potiers décorateurs de ce groupe, acquiert vraisemblablement son autonomie en fondant sa propre officine. L'abandon de la marque cursive et des petites estampilles au profit désormais d'une seule marque de grand format, dépourvue de cartouche $(52 \mathrm{~mm})$, est un indice

\footnotetext{
255. Sur un total de 99 décors avec marque épigraphique appartenant à la période d'activité " classique " de l'officine de Paternus ("Paternus IIb»: 170-début du III $^{\mathrm{E}}$ s.), $94 \%$ portent l'estampille PATER $\overline{\text { FE }}$ (Delage, 1999a, vol. 4, p. 39). Notons, par ailleurs, que deux ou trois mentions seulement de graffites in forma en position infradécorative ont été relevées sur les décors du groupe Paternus postérieurs à 160 . Ils constituent souvent des abréviations à usage " interne ".

256. La multiplication, après 170 , du nombre d'oves et de lignes-sousoves sur les décors de Paternus est, en l'absence de marque nominative, un indice relativement fiable de la multiplicité des individus ayant œuvré au sein de cette officine ou d'autres unités de production.
}

257. À savoir fondée sur un échantillon dont la représentativité a été validée, ainsi qu'une démarche reposant sur des quantifications (Delage, 1999b).

258. Famille précoce dite "Cinnamus A " par G. B. Rogers (1999, p. 135) ou "Cinnamus I" par A.-.M. Romeuf (2001, p. 113). La dénomination et la démarche méthodologique de cette dernière doivent être privilégiées. La datation proposée reste toutefois trop large (jusqu'aux années 180). Dans ce domaine, la proposition de G. Rogers paraît plus acceptable : 135-160.

259. De graphie OFISACRI, longue de $43 \mathrm{~mm}$ (Simpson, Rogers, 1969, fig. $2, n^{\circ} 7$; Stanfield, Simpson, 1990, pl. 83, n8). 

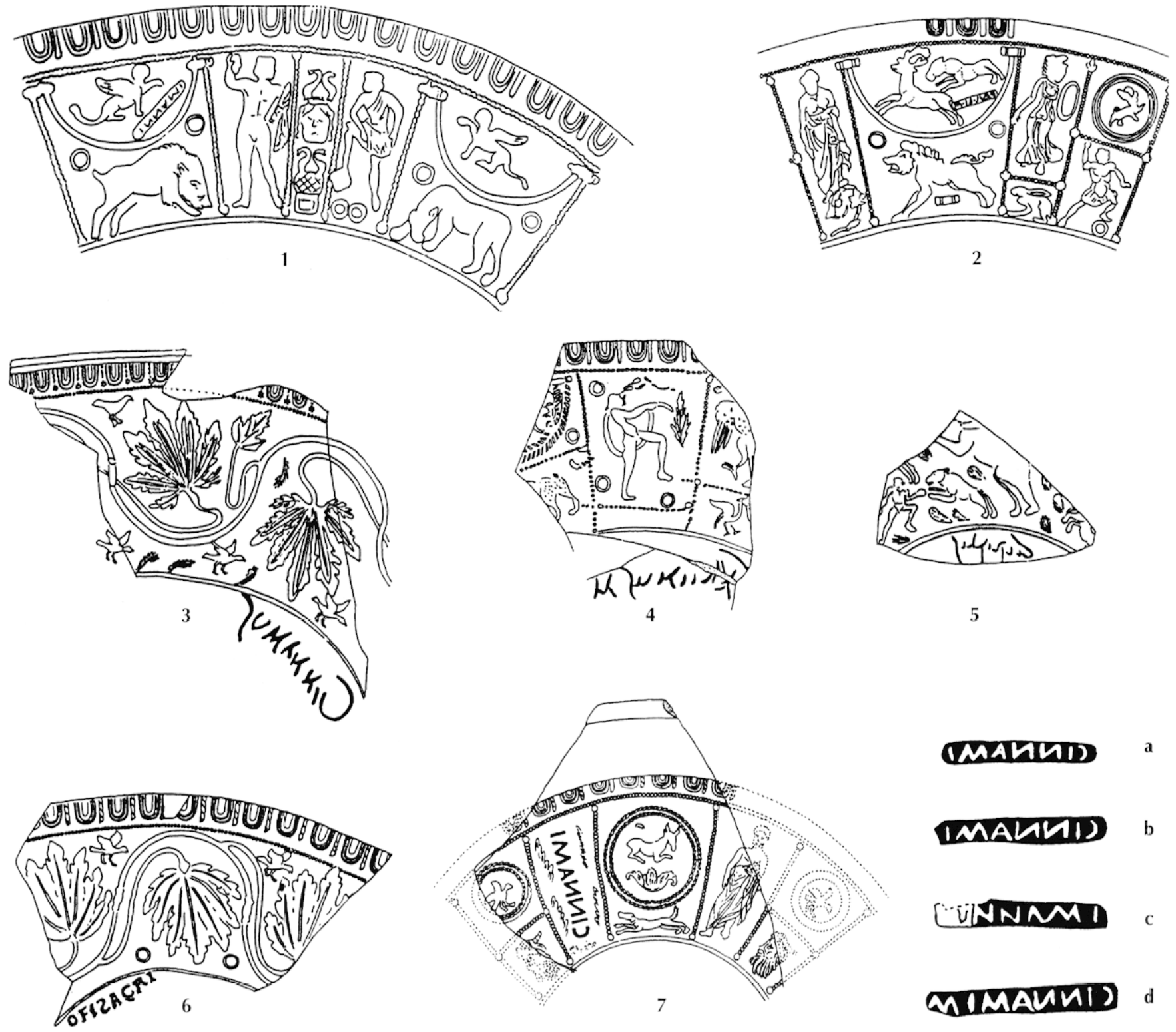

(MARиा)

a

IMALИ1) b

\section{UNNNI}

momavinis d

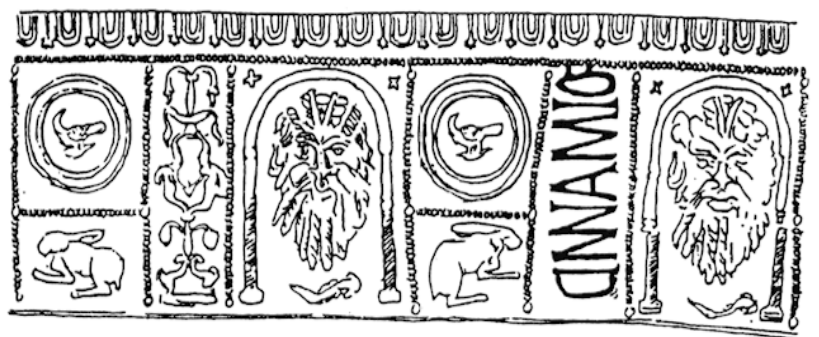

IMAИИIS.

$60 \mathrm{~mm}$

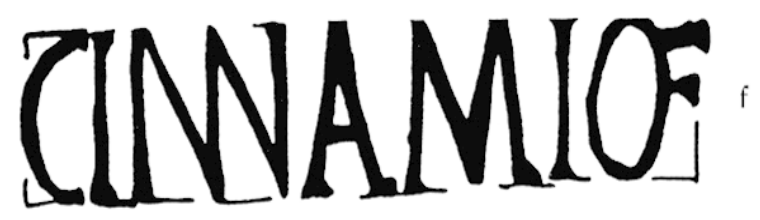

0

$20 \mathrm{~mm}$

Fig. 78 - Marques intradécoratives au nom de Cinnamus (a-f, cf. Romeuf-Vialatte, 1970) et décors avec marque épigraphique du groupe Cinnamus : $\boldsymbol{a}, \boldsymbol{b}$, e, estampilles rétrogrades de graphie CINNAMI; c, estampille antégrade de graphie [CIINNAMI; d, estampille rétrograde de graphie CINNAMIM ; f, estampille antégrade de graphie CINNAMI $\overline{O F} ; 1$, vase moulé comportant une petite estampille intradécorative rétrograde de graphie CINNAMI (estampille b), provenance : Étaples, Pas-de-Calais (Piton, 1985, pl. 13, $n^{\prime \prime} 3$ ) ; 2, vase moulé comportant une petite estampille intradécorative rétrograde de graphie PAVL.LI.M, provenance: Wroxeter, Shropshire, Grande-Bretagne (Stanfield, Simpson, $\left.1990, p l .165, n^{\circ} 1\right) ; 3$, vase moulé comportant une marque cursive infradécorative rétrograde de graphie Cinnamus, provenance : Chester, Cheshire, Grande-Bretagne (Stanfield, Simpson, 1990, pl. 162, $n^{\circ} 57$ ) ; 4, vase moulé comportant une marque cursive infradécorative rétrograde de graphie Galiinus M, provenance : Les Martres-de-Veyre, Puy-de-Dôme (Romeuf, Romeuf, 2001, pl. 108, $\left.n^{\circ} 2\right)$; 5, vase moulé comportant une marque cursive infradécorative rétrograde de graphie Ciirialis, provenance: Amiens, Somme (Rogers, 1999, pl. 29, $\left.n^{\prime \prime} 4\right)$; 6, vase moulé comportant une estampille infradécorative antégrade de graphie OFISACRI, provenance : Corbridge, Northumberland, GrandeBretagne (Stanfield, Simpson, 1990, pl. 83, $n^{\circ} 8$ ) ; 7, vase moulé comportant une estampille intradécorative rétrograde de graphie CINNAMI (estampille e), provenance: Hallstatt, Haute-Autriche (Karnitsch, 1959, pl. 75, $n^{\circ} 1$ ); 8, moule comportant une estampille intradécorative antégrade CINNAMIOF (estampille f), provenance : Terre-Franche à Bellerive-sur-Allier; Allier (Vauthey, Vauthej, 1993, p. 41, fig. 28). 
révélateur de ce changement (fig. 78e, n7). L'activité et la rentabilité de cette nouvelle officine sont probablement sans commune mesure avec ce qu'elles furent précédemment. Les millions de vases moulés portant un décor de Cinnamus diffusés dans le monde romain occidental en l'espace de quelques décennies impliquent nécessairement une production de moules intensive, des réseaux bien structurés de distribution de ces outils et la création de "succursales " au sein d'autres centres de production de la vallée de l'Allier, comme l'a suggéré A.-M. Romeuf dans sa thèse ${ }^{260}$. Là encore, comme dans le cas du groupe Paternus, tous les artisans œuvrent dans le même sens, la promotion des ateliers. Il n'est pas inintéressant, d'ailleurs, de noter que les succursales de Terre-Franche/Lubié adoptent une nouvelle estampille, encore plus grande que la précédente $(67 \mathrm{~mm})$, de graphie CINNAMIOF (fig. $\left.78 \mathrm{f}, \mathrm{n}^{\circ} 8\right)$.

\section{UN RÔLE À JOUER DANS LA STRATÉGIE COMMERCIALE}

Les schémas d'évolution de ces deux groupes stylistiques présentent de fortes similitudes. Lors des phases précoces, celles du deuxième quart $\mathrm{du} \mathrm{II}^{\mathrm{c}} \mathrm{s}$., les coutumes et savoir-faire en matière de décoration semblent exclure toute "fantaisie " en ce qui concerne les marques intradécoratives. Les potiers utilisent des petites estampilles apposées dans les espaces laissés libres entre les poinçons ou, plus exceptionnellement, en remplacement d'un motif secondaire. Ceux, en revanche, qui apposent leur nom sous le décor disposent de plus de liberté, ce qui se traduit par des libellés de taille très variable : des cursives, à l'écriture ample d'au moins $40 \mathrm{~mm}$, aux petites estampilles. Cette absence de contrainte est due au fait que ces marques sont susceptibles d'être effacées par les potiers tourneurs ${ }^{261}$, mais aussi qu'elles apparaissent sur une surface vierge de tout décor, incapable de dénaturer une composition

260. Voir Vialatte, 1968 , p. 8-11 et 150 . Des officines concevant des moules du groupe Cinnamus ont existé à Lezoux ainsi qu'à Lubié/Terre-Franche. Les caractéristiques stylistiques des productions de ces deux pôles sont suffisamment éloignées pour que cette hypothèse puisse être validée. En revanche, l'existence d'une officine aux Martres-de-Veyre est sujette à caution. La présence, en grand nombre, de vases moulés ou même de moules ne peut constituer un indice de production locale, puisque les compositions attestées sont similaires à celles des ateliers lézoviens. De ce fait, les moules utilisés par les potiers locaux ont vraisemblablement été importés (voir sur ces questions de localisation des officines de sigillées moulées: Delage, 2000, p. 117-120): Ainsi en va-t-il des découvertes effectuées à Toulonsur-Allier et Manglieu (Bet et al., 2000) ou même Banassac (Rogers, 1970 ; Morel, Peyre, 1973).

261. Le potier tourneur est celui qui se sert de la matrice pour fabriquer le vase moulé. Sur le plan technique, son travail ne s'inscrit pas nécessairement dans la continuité des étapes précédentes, puisque le moule est un outil fini qui peut être cédé à n'importe quelle officine. Pour concevoir un Drag. 37 de l'époque de Cinnamus et Paternus, le potier tourne le corps principal du vase au sein du moule et poursuit son action jusqu'à concevoir le bandeau supérieur en dehors de celui-ci ; puis il façonne, à part, un pied qu'il applique au fond du vase après ou d'altérer sa structure répétitive. Toutes servent ainsi essentiellement à contrôler et organiser l'activité de production.

Les secondes phases d'évolution de ces groupes, à partir de 150-160, révèlent un changement très net : la marque tend désormais à s'afficher (existence d'une phase de transition pour Paternus). Elle devient non pas la «signature " d'un potier décorateur, mais une " raison sociale ". Elle perd ainsi complètement son caractère individuel, mais aussi, en grande partie, son rôle dans l'organisation du travail au profit d'une fonction " commerciale».

La bonne lisibilité des estampilles de grand format, du fait de leur taille ${ }^{262}$, l'usage que les potiers en font, ainsi que leur présence sur près d'un tiers des moules selon certaines estimations, le démontrent bien. Il ne fait guère de doute que potiers et autres intervenants impliqués dans la chaîne opératoire de production/diffusion ont désiré créer des " labels " en quelque sorte, pour faciliter la vente, maintenir leurs acquis face à la concurrence, "fidéliser la clientèle " et assurer ainsi la renommée de leurs produits. La conservation du nom Cinnamus pour les succursales de Terre-Franche/Lubié montre que les potiers revendiquent leur filiation avec l'officine mère de Lezoux, espérant ainsi probablement retirer les bénéfices d'un " label " désormais connu de tous. En définitive, chacun y trouve son compte : les officines mères ou filiales qui pérennisent leurs activités, les potiers tourneurs qui conçoivent des vases moulés ayant plus de valeur ajoutée que les autres ${ }^{263}$, les vendeurs qui disposent d'un argument commercial et les acheteurs qui peuvent s'enorgueillir de posséder un "Cinnamus" 264.

Précisons que ces deux groupes de production ne sont pas les seuls à participer à ce mouvement et qu'il est possible qu'ils n'en soient pas les initiateurs, mais plutôt les plus grands utili-

démoulage. C'est à la suite de cette dernière action que les marques sous le décor sont susceptibles de disparaître. En effet, pour assurer la cohérence entre les parties, ainsi qu'une finition de qualité, le potier lisse la zone entre le pied et le décor. Les marques étant en relief, elles s'effacent au même titre que les autres imperfections.

262. La présence de nombreuses ligatures au sein de l'estampille PATERNVS ne devait pas constituer un handicap à sa lecture, car ce type de pratique était couramment répandu dans l'Antiquité.

263. L'intérêt que présentent certains moules aux ycux des potiers tourneurs se marque par leur conservation à plus long terme, y compris lorsque l'usure de l'outil a altéré considérablement la finesse et la lisibilité du décor. Les vases issus de ces moules « résiduels " présentent des qualités techniques très différentes de celles des pièces contemporaines de la période standard de fabrication/diffusion/utilisation des outils. Parmi les officines concernées par ce phénomène, celles de Cinnamus et surtout Paternus figurent en bonne place. Cela est tout particulièrement le cas pour cette dernière, puisque de nombreux vases moulés sont présents dans les niveaux du $\mathrm{III}^{\mathrm{e}} \mathrm{s}$. Voir par exemple le contexte US 1007 de la fouille de la place des Halles au Mans, datant de la fin du Haut-Empire (Delage, Guillier, 1997, p. 271) ou bien encore celui de la boutique de la fouille du couvent des Cordeliers à Poitiers, du milieu du $\operatorname{III}^{\mathrm{e}} \mathrm{s}$. (Wittmann, Jouquand, à paraître).

264. Est-ce le cas de ce soldat de Flavia Solva, un camp militaire du Norique, qui a inscrit sous le pied de son vase moulé portant un décor de Cinnamus: "Coupe de Verecundus achetée pour 20 as "? (Noll, 1972). 
sateurs, ceux qui lui ont véritablement donné un sens ${ }^{265}$. Il est intéressant, également, de noter que les autres marques intradécoratives de grand format les plus anciennes, celles du milieu du II $^{\mathrm{e}}$ s., comportent fréquemment une mention d'officine : OF.ATT ${ }^{266}$, OFISACRI ${ }^{267}$, mais aussi des libellés au nom de Q.I.BALBINVS. Elles renforcent ainsi l'idée qu'elles ont été créées, avant tout, pour représenter des collectifs. Parmi les autres mentions, citons MACCIVS, CATVSSA II, SERVVS II, ANTISTII, ainsi que BANVVS I et BANVVS II (dont la plus grande atteint presque $80 \mathrm{~mm}$ de long !), toutes de la seconde moitié du $\mathrm{II}^{\mathrm{e}} \mathrm{s}$. Sauf exceptions, elles caractérisent de petites unités de production, parfois fort méconnues tant le nombre de décors recensés s'avère faible. Pour ces officines, l'usage des marques de grand format est le signe avant tout de leur alignement sur les pratiques des ateliers dominants.

Mentionnons, enfin, le fait que les concurrents les plus importants des ateliers du Centre de la Gaule ne sont pas restés sans réponse face à ces nouvelles pratiques. Certaines officines alsaciennes (Heiligenberg essen- tiellement), ainsi que celles de Rheinzabern qui, au $\mathrm{II}^{\mathrm{C}}$ s., disputent aux vases arvernes les marchés du plateau suisse et des provinces danubiennes ${ }^{268}$,

265. Notons qu'avant le milieu du II" s., quelques officines phares s'étaient timidement affranchies du format des petites estampilles en mettant en valeur leur marque intradécorative par l'adoption notamment d'une graisse "typographique " plus importante. Tel est le cas de Titos pour le Irrs. (Piboule et al., 1981), de Libertus I dont l'une des estampilles "OFILIBERTI " mesure $35 \mathrm{~mm}$ sans cartouche, ou encore de Butrio dont les marques, sans être plus longues que les petits formats, sont plus hautes. L'estampille AVSTRI.OF appartient également à cette catégorie intermédiaire. Flle présente une longueur de $42 \mathrm{~mm}$ tout en conservant un cartouche. Cet exemple demeure, toutefois, particulier. Fon effet, des ateliers créateurs de vases moulés dans le style "d'Austrus" existent aussi bien à lezoux (province d'Aquitaine) qu'à Blickweiler (province de Gaule belgique). Sur ces deux sites, aucune marque AVSTRI.OF n'a été retrouvée parmi les lots issus des dépotoirs, si bien qu'un doute subsiste sur son origine précise, mais aussi, par extension, sur sa période d'utilisation. Cette estampille n'est, en effet, pas nécessairement contemporaine de la petite marque intradécorative AVSTRI.M., que l'on rencontre sur les moules et vases moulés lézoviens (Pferdehirt, 1987 ; Chuniaud, 2003, p. 248).

266. La datation proposée par Stanfield, Simpson 1990 et Rogers 1999 pour les décors portant cette marque (125-150) est trop précoce car fondée sur très peu d'indices. Les découvertes lézoviennes permettent plutôt d'envisager une activité au milieu du II" s.

267. Cette marque est identique à celle que l'on retrouve associée à la phase précoce de Cinnamus. En position intradécorative, elle apparaît toutefois sur des décors d'un autre groupe de production : Attianus II / Sacer II / Criciro (Stanfield, Simpson, 1990, pl. 82, n"5).

268. L.es productions d'Heiligenberg sont diffusées dans la vallée du Rhin supérieur, ainsi que sur le plateau suisse, dès le deuxième quart du adoptent, elles aussi, de telles marques. Si peu d'estampilles s'affranchissent des cartouches, bon nombre d'entre elles atteignent tout de même $40 \mathrm{~mm}$ de long, pour des hauteurs pouvant dépasser $10 \mathrm{~mm}$. Le fait qu'elles soient, en grande partie, contemporaines de celles de Cinnamus et Paternus n'est pas un hasard. La réactivité des ateliers est souvent telle que les innovations les plus pertinentes des uns sont bien souvent mises en œuvre par les autres dans un délai très court ${ }^{269}$.

\section{UNE FIN ANNONCÉE}

La " mode " des marques de grand format occupant une place bien visible dans la composition des décors disparaît avec le retrait définitif des vaisselles sigillées du Centre de la Gaule, au début $\mathrm{du} \mathrm{III}^{\mathrm{e}} \mathrm{s}$., de tous les marchés à longue distance. Une des raisons principales n'est guère difficile à appréhender. Elle tient à l'affaiblissement des enjeux commerciaux, puisque les volumes de production sont désormais bien plus faibles et qu'au sein des régions approvisionnées (Gaule lyonnaise et nord de la province d'Aquitaine) n'existe pas de véritable concurrence en ce qui concerne les vaisselles de table (Delage, 1998). Il n'est donc plus nécessaire (ou moins nécessaire) de mettre en ouvre de véritables stratégies commerciales dont l'écrit est un des maillons.

II" s. Flles constituent pour les utilisateurs helvètes une alternative aux vaisselles du Centre de la Gaule jusqu'à la fin du II"s. Leur commercialisation au sein des provinces danubiennes demeure très restreinte, car la capacité de production des ateliers alsaciens, particulièrement lorsque apparaissent ceux de Rheinzabern, est trop faible pour remplir les conditions d'une présence viable sur les marchés à longue distance. Les ateliers de Rheinzabern ne débutent, quant à eux, leur activité qu'au milieu du II $^{\prime \prime} s$. S'ils conquièrent rapidement une partie des marchés de Germanie, ils mettent, en revanche, plus de temps à remporter ceux du plateau suisse et des provinces danubiennes, en raison de la bonne implantation des vaisselles du Centre de la Gaule. C'est à la fin du troisième quart du II' $s$. que les proportions s'inversent entre les deux pôles de production, les diffuseurs des vases arvernes abandonnant alors progressivement ces territoires.

269. Les quelques estampilles sur sigillée moulée de Rheinzabern, dépourvues de cartouche, appartiennent toutes à la première phase d'activité des ateliers du milieu et de la seconde moitié du II"s. L.es marques de Colmertus $I$, $I I$ et $I I I$, par exemple, dépassent les $50 \mathrm{~mm}$ de long pour 6 à $7 \mathrm{~mm}$ de haut et se trouvent régulièrement mises en valeur au sein des décors (Ricken, 1948, pl. 22, nº $10 ;$ pl. 23, nº $1 ;$ pl. 24 , $n^{\prime \prime} 4 f$; pl. 25, n"1, etc.). Les grandes estampilles à cartouche appartiennent également, dans leur majorité, à l'activité du II‘s. (Kortüm, Mees, 1998). Les officines de Cerialis I, Firmus I, B.FAttomi utilisent toutes des estampilles de plus de $40 \mathrm{~mm}$ de long pour 8 à $10 \mathrm{~mm}$ de haut. 